\title{
Measurement of the SM Higgs boson mass in the diphoton and $4 \ell$ decay channels using the ATLAS detector
}

Karolos Potamianos*†

Deutsches Elektronen-Synchrotron (DESY)

E-mail: karolos.potamianos@cern.ch

\begin{abstract}
One of the fundamental properties of the Higgs boson, its mass, is measured by studying the invariant mass of the $4 \ell$ and diphoton decay channels with about $36 \mathrm{fb}^{-1}$ of data collected by the ATLAS experiment at the Large Hadron Collider (LHC). Since in these channels the final state can be fully reconstructed, an invariant mass peak with a good experimental resolution can be obtained in a model independent way and used as an input to compare other Higgs properties measurements with the SM predictions. The measured values of the Higgs boson mass for the $H \rightarrow Z Z^{*} \rightarrow 4 \ell$ and $H \rightarrow \gamma \gamma$ decay modes are $m_{H}=124.88 \pm 0.37$ (stat) \pm 0.05 (syst) GeV and $m_{H}=125.11 \pm 0.21$ (stat) \pm 0.36 (syst) GeV respectively, and the mass derived from the combined fit is $m_{H}=124.98 \pm 0.19$ (stat) \pm 0.21 (syst) GeV. These results are in good agreement with the LHC Run-1 average of $125.09 \pm 0.24 \mathrm{GeV}$.
\end{abstract}

The European Physical Society Conference on High Energy Physics

5-12 July

Venice, Italy

\footnotetext{
* Speaker.

${ }^{\dagger}$ On behalf of the ATLAS collaboration
} 


\section{Introduction}

The observation of a new particle in the search for the Standard Model (SM) Higgs boson $(H)$ by the ATLAS and CMS experiments $[1,2]$ at the Large Hadron Collider (LHC), using Run-1 proton-proton $(p p)$ collision data at center-of-mass energies of $\sqrt{s}=7 \mathrm{TeV}$ and $8 \mathrm{TeV}$, was a major step in understanding the mechanism of electroweak symmetry breaking (EWSB) [3, 4, 5]. The mass of the Higgs boson, $m_{H}$, has been measured to be $125.09 \pm 0.24 \mathrm{GeV}$ [6] through a combination of the individual ATLAS [7] and CMS [8] measurements with Run-1 data. Recently, the CMS collaboration measured the Higgs boson mass in the $H \rightarrow Z Z^{*} \rightarrow 4 \ell$ channel using $35.9 \mathrm{fb}^{-1}$ of $p p$ collision data at $13 \mathrm{TeV}$ [9].

We report a measurement of the Higgs boson mass with $36.1 \mathrm{fb}^{-1}$ of $\sqrt{s}=13 \mathrm{TeV} p p$ collision data recorded with the ATLAS detector [10]. This measurement is derived from a combined fit to the invariant mass spectra $m_{4 \ell}$ and $m_{\gamma \gamma}$ of the decay channels $H \rightarrow Z Z^{*} \rightarrow 4 \ell(\ell=e, \mu)$ and $H \rightarrow \gamma \gamma$.

\section{The ATLAS experiment}

The ATLAS experiment [11] at the LHC is a multi-purpose particle detector with nearly $4 \pi$ coverage in solid angle. It consists of an inner tracking detector (ID) surrounded by a $2 \mathrm{~T}$ superconducting solenoid, electromagnetic and hadronic calorimeters, and a muon spectrometer (MS) incorporating three large superconducting toroidal magnet systems. The ID provides tracking for charged particles for $|\eta|<2.5$. The calorimeter system covers the pseudorapidity range $|\eta|<4.9$. The MS includes both high-precision tracking chambers $(|\eta|<2.7)$ and fast trigger chambers $(|\eta|<2.4)$. On-line event selection is performed by a first-level trigger with a maximum output rate of $100 \mathrm{kHz}$, implemented in custom electronics, followed by a software trigger with a maximum output rate of $1 \mathrm{kHz}$.

\section{Muon, photon and electron reconstruction, identification and calibration}

Muon track reconstruction is first performed independently in the ID and the MS. Hit information from the individual sub-detectors is then used in a combined muon reconstruction, which includes information from the calorimeters. Although the simulation accurately describes the ATLAS detector, additional corrections to the reconstructed momentum are needed in order to match the simulation to data precisely [12]. The muon momentum resolution and momentum scale are parameterised as a power expansion of the muon $p_{T}$, as a function of $\eta$ and $\phi$ from large samples of $J / \Psi \rightarrow \mu^{+} \mu^{-}$and $Z \rightarrow \mu^{+} \mu^{-}$decays. The momentum scale is known to a precision of half per mille for muons with $|\eta|<1.5$ and about four per mille for $|\eta|>2.0$. The data taken in 2016 were affected by a misalignment of parts of the ID. This causes a bias on the measured momentum of each muon which is corrected by an iterative procedure derived from $Z \rightarrow \mu^{+} \mu^{-}$decays in data. From percent-level dimuon mass $\left(m_{\mu \mu}\right)$ bias, the residual effect after the correction is reduced to the per-mille level at the scale of the $Z$ boson mass, $m_{Z}$. The uncertainty associated with this correction is about $20 \mathrm{MeV}$ for the average momentum of muons from $Z \rightarrow \mu^{+} \mu^{-}$decays.

Photon and electron candidates are reconstructed from clusters of energy deposited in the electromagnetic (EM) calorimeter. Clusters without a matching track or reconstructed conversion vertex in the ID are classified as unconverted photons. Those with a matching reconstructed 
conversion vertex or a matching track consistent from originating from a photon conversion are classified a converted photons [13]. Clusters matched to a track consistent with originating from an electron produced in the beam interaction region are considered electron candidates. The energy measurement for reconstructed electrons and photons is performed by summing the energies measured in the EM calorimeter cells belonging to the candidate cluster. The different layers of the EM calorimeter are intercalibrated using the result obtained in Ref. [13] (with uncertainties to cover possible changes in the 2015 and 2016 data compared to that of 2012). The cluster energy is corrected for energy loss in the inactive materials in front of the calorimeter, the fraction of energy deposited outside the area of the cluster in the $\eta-\phi$ plane, for the amount of energy lost behind the EM calorimeter, and to account for the variation of the energy response as a function of the impact point in the calorimeter. The detector response, obtained from a detailed simulation, is calibrated separately for electrons, converted and unconverted photon candidates. The global calorimeter energy scale is determined in situ with a large sampled of $Z \rightarrow e^{+} e^{-}$events. The energy response in data and simulation is equalised by applying $\eta$-dependent calibration factors for data to match the invariant mass distribution of $Z \rightarrow e^{+} e^{-}$events.

\section{Mass measurement in the $H \rightarrow Z Z^{*} \rightarrow 4 \ell$ channel}

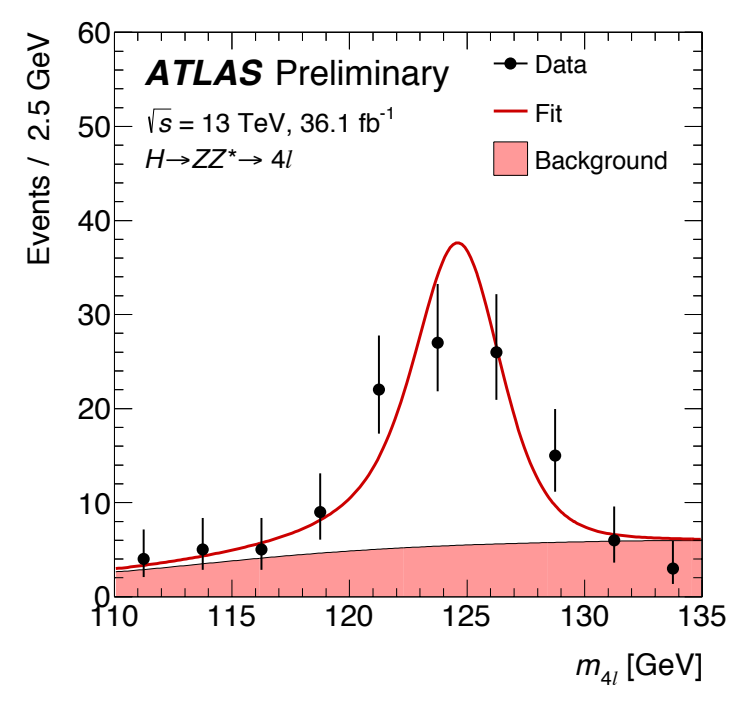

(a)

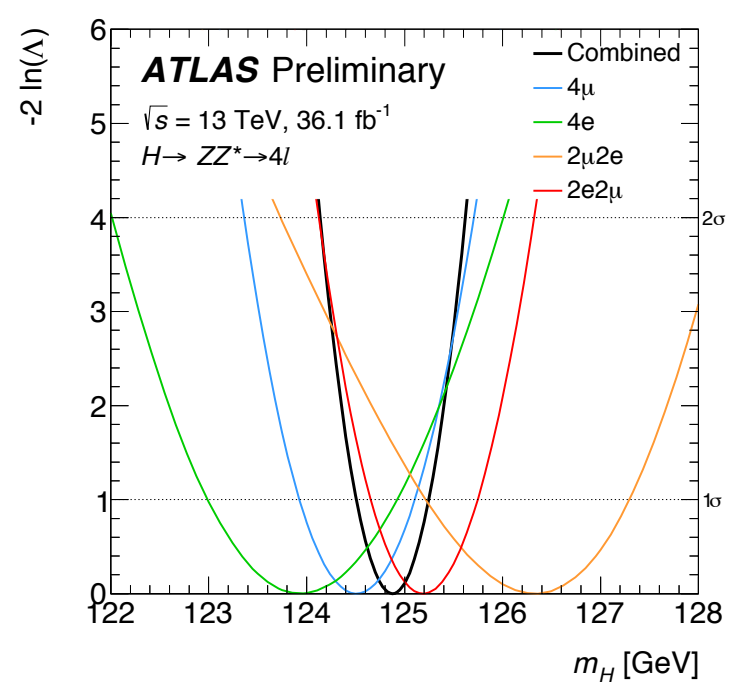

(b)

Figure 1: (a) Invariant mass distribution of the data (black points with error bars) shown with the projection of the simultaneous fit result to $H \rightarrow Z Z^{*} \rightarrow 4 \ell$ candidates (continuous line). The background component of the fit is also shown (filled area). The signal pdf is evaluated per-event and averaged over the observed data. (b) Value of $-2 \ln \Lambda$ as a function of $m_{H}$ for the combined fit and for the measurements in the individual lepton-decay channels [10].

The selected events are required to contain at least four isolated leptons $(\ell=e, \mu)$ that are reconstructed at a common vertex and form two oppositely charged pairs of same flavour. The 4 leptons, ordered by decreasing transverse momentum $p_{T}$, are required to have a $p_{T}$ greater than (in 
$\mathrm{GeV}) 20,15,10$, respectively for the first to third lepton, and $5(\mu)$ or 7 (e) for the fourth lepton. The latter was relaxed compared to the Run- 1 analysis, and a $4 \ell$ vertex cut has been added to counter-balance the increase of reducible background caused by the reduction in the $p_{T}$ threshold.

The lepton pair with an invariant mass closest (second closest) to the $Z$ boson pole mass in each quadruplet is referred to as the leading (subleading) dilepton pair. The selected events are split according to the flavour of the leading and subleading pairs; ordered according to the expected selection efficiency, they are $4 \mu, 2 e 2 \mu, 2 \mu 2 e, 4 e$. If more than four leptons are found in the event, only the quadruplet with the largest expected efficiency is kept. Finally, reconstructed photon candidates passing final-state radiation selections are combined with the lepton quadruplet, and a kinematic fit is performed to constrain the invariant mass of the leading lepton pair to the $Z$ pole mass [7], which improves the $m_{4 \ell}$ resolution by $15 \%$.

In the fit range of $110<m_{4 \ell}<135 \mathrm{GeV}, 122$ candidate events are observed. Non-resonant $Z Z^{*}$ production with a final state topology similar to the signal is the main background contribution ( $84 \%$ of the total background). It is estimated using simulation normalised to the best available predictions and validated in the sidebands of the selected mass range. The remaining backgrounds, $Z+$ jets, $W Z$ and $t \bar{t}$, where one or more hadrons or their decay products are misidentified as a prompt lepton, are estimated from data using minimal input from simulation, as described in Ref. [14]. Other backgrounds are $t \bar{t}+Z, Z Z Z, W Z Z, W W Z$, and account for less than $1 \%$ of the total background. They are estimated from simulation.

The Higgs boson is more centrally produced than the non-resonant $Z Z^{*}$ production and tends to have larger transverse momentum. To improve the sensitivity, the events are further split into four exclusive equal-size bins of a Boosted Decision Tree (BDT), using the following inputs: the transverse momentum and pseudorapidity of the four-lepton system and a matrix-element-based kinematic discriminant $D_{Z Z^{*}}=\ln \left(\left|\mathscr{M}_{H Z Z *}\right|^{2} /\left|\mathscr{M}_{Z Z}\right|^{2}\right)$ [15], where $\mathscr{M}_{X}$ are matrix elements calculated with MADGRAPH5 [16].

The measured $m_{4 \ell}$ signal distribution is modelled as the convolution of the intrinsic Higgs boson decay, assumed to be a relativistic Breit-Wigner (BW) distribution with width equal to $4.1 \mathrm{MeV}$ and a pole at $m_{H}$, with a four-lepton invariant mass response function defined as the probability of measuring a value $m_{4 \ell}$ for a true invariant mass $m_{4 \ell}^{\text {true }}$. The $m_{4 \ell}$ response function is derived on an event-by-event basis with the following procedure. The lepton energy response distribution is first modelled in simulation with a weighted sum of 3 Gaussian distributions. The $m_{4 \ell}$ response is expressed as a convolution of $3^{4}=81$ Gaussian distributions. The probability density function (pdf) is then simplified using a merging method: at each step, Gaussian pdf are compared using the Kullback-Leibler distance [17] and the closest pair is merged into a single Gaussian (with parameters derived in a manner that preserves the first two moments of the original distribution). The procedure is repeated until the pdf consists of a linear combination of four Gaussians (the minimal set found to preserve the initial information). More details on the procedure are given in Ref. [10]. Residual differences to the true lepton energy are incorporated into the model by rescaling the parameters of the reduced response function to reproduce the four-lepton signal invariant mass distribution in simulation. A validation with data is performed with $Z \rightarrow 4 \ell$ events (setting the pole and width of the BW distribution to those of the $Z$ boson). The measured values agree with the world average of the $Z$-boson mass within less than 1.3 standard deviations in data. As an cross-check, a template-based method [7] is also used to measure $m_{H}$. 


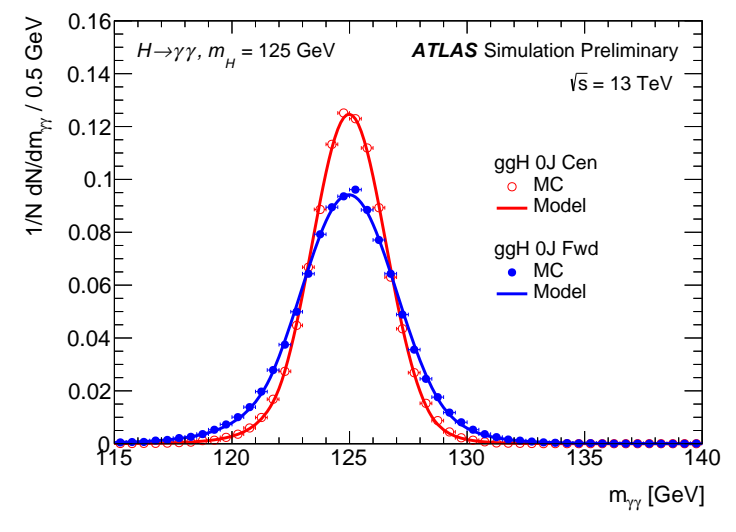

(a)

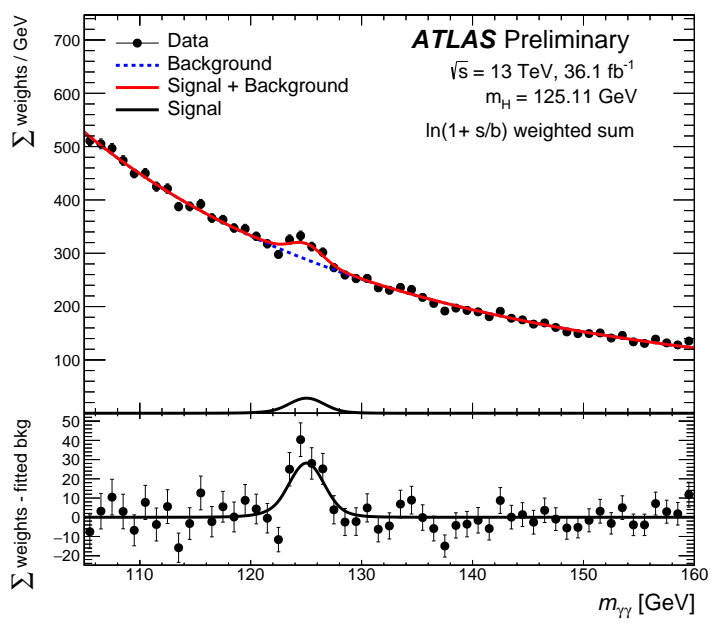

(b)

Figure 2: (a) Invariant mass distribution (circles) of simulated $H \rightarrow \gamma \gamma$ events reconstructed in two categories with one of the best ("ggH 0J Cen": open circles) and one of the worst (" $g g H 0 \mathrm{~J}$ Fwd": solid circles) experimental resolutions. The signal model derived from a fit of the simulated events is superimposed (solid lines). (b) Diphoton invariant mass distribution of the data superimposed with the result of the fit. Both for data and the fit each category is weighted by a factor $\log (1+s / b)$. The dashed line represents the background component of the model, while the black line the signal component. The bottom inset is the difference between the sum of weights and the background component of the fitted model [10].

The estimate of $m_{H}$ for the per-event and template methods is extracted with a simultaneous profile likelihood fit to the sixteen categories. The measured value of the mass of the Higgs boson is found to be

$$
m_{H}=124.88 \pm 0.37 \text { (stat) } \pm 0.05 \text { (syst) GeV. }
$$

Figure 1 shows the invariant mass distribution of the data together with the value of $-2 \ln \Lambda$ as a function of $m_{H}$ for the combined fit and for the measurements in the individual lepton-decay channels $(4 \mu, 4 e, 2 \mu 2 e, 2 e 2 \mu)$. The observed total uncertainty in $m_{H}$ for the per-event method is of $\pm 0.37 \mathrm{GeV}$. For the template method it is found to be ${ }_{-0.40}^{+0.41} \mathrm{GeV}$. The observed difference for the $m_{H}$ estimates of the two methods is found to be $0.16 \mathrm{GeV}$, which is compatible with the expected variance estimated with pseudo-experiments and corresponds to a one sided p-value of 0.19. The total systematic uncertainty is $47 \mathrm{MeV}$, with the leading sources being the muon momentum scale $(40 \mathrm{MeV})$, the electron energy scale $(20 \mathrm{MeV})$, the background modeling (10 MeV) and the simulation statistics $(8 \mathrm{MeV})$.

\section{Mass measurement in the $H \rightarrow \gamma \gamma$ channel}

Following an initial pre-selection, requiring to have at least two loosely-identified photon candidates with $E_{T}>25 \mathrm{GeV}$ and $|\eta|<2.37$ (excluding the range $1.37 \leq|\eta| \leq 1.52$ ), events are selected if the leading (subleading) photon candidates have $E_{T} / m_{\gamma \gamma}>0.35(0.25)$. Only events with $105 \leq m_{\gamma \gamma} \leq 160 \mathrm{GeV}$ are kept. The events are then classified, based on the properties of the 
two selected photons and of jets, $b$-jets, electrons, muons and missing transverse momentum, in 31 mutually exclusive categories.

In the diphoton channel, the Higgs boson mass is measured from the position of the narrow resonant peak in the $m_{\gamma \gamma}$ distribution, observed on top of a large, monotonically decreasing, distribution from continuum background events. For each category, the shape of the $m_{\gamma \gamma}$ distribution of the signal is modelled with a double-sided Crystal Ball function [18], i.e. a Gaussian function in the peak region with power-law functions in both tails. The background invariant mass distribution of each category is parameterised with an empirically chosen continuous function, whose parameters are fitted directly on data. The functional form is chosen among several alternatives according to three criteria [16]: the fitted signal yield on a test sample representative of the background (built by combining simulation and control regions in data) must be minimized; the $\chi^{2}$ probability for the fit of the background control sample must be larger than a certain threshold; and the fit quality to data sidebands must not improve significantly when adding an extra degree of freedom. The models selected by this procedure are exponential distributions or power-law functions with one degree of freedom for categories with less events, while exponential functions of a second-order polynomial are used for the others. The Higgs boson mass in the diphoton channel is estimated with a simultaneous binned maximum likelihood fit to the $m_{\gamma \gamma}$ distributions of the selected event categories. The parameters of the fit are $m_{H}$, the four signal strengths, the number of background events, the parameters describing the shape of the background invariant mass distribution and all the nuisance parameters associated to systematic uncertainties.

The measured Higgs boson mass in the diphoton channel is

$$
m_{H}=125.11 \pm 0.21 \text { (stat) } \pm 0.36 \text { (syst) GeV }
$$

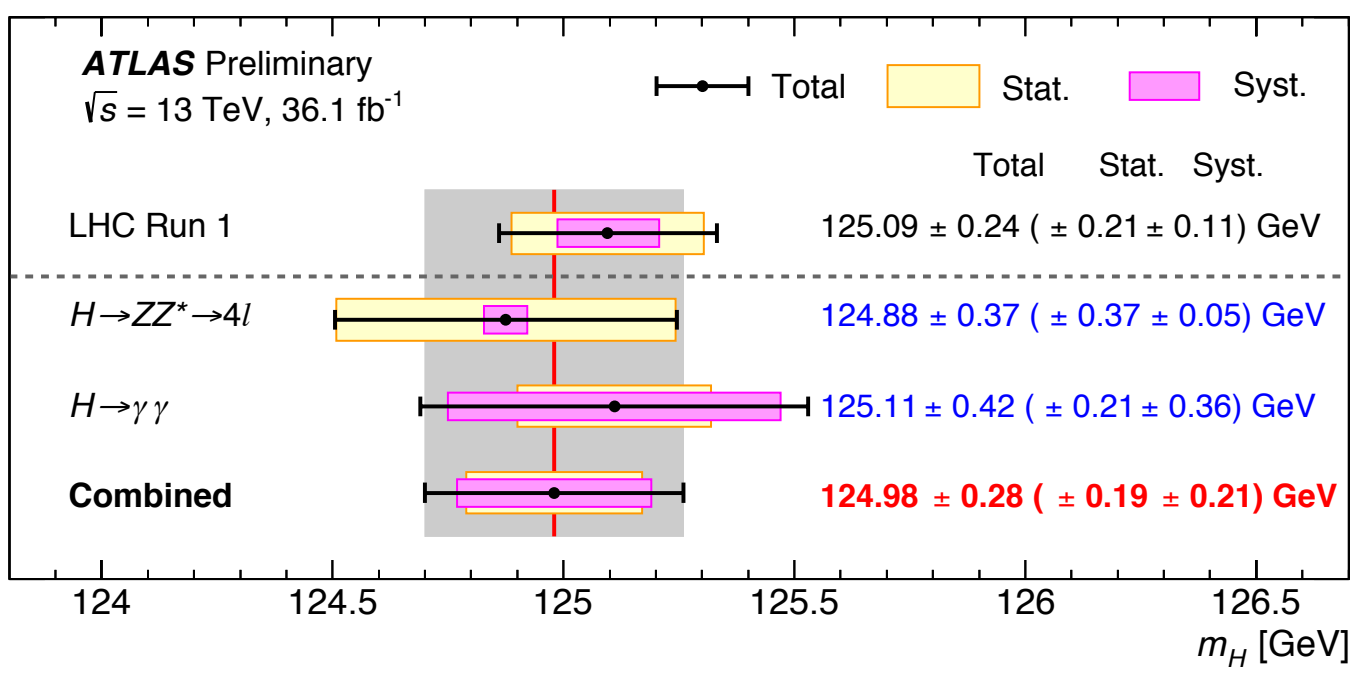

Figure 3: Summary of the Higgs boson mass measurements from the individual analyses presented here, as well as their combination, compared to the combined Run-1 measurements by ATLAS and CMS [6]. The systematic (magenta-shaded bands), statistical (yellow-shaded bands), and total (black error bars) uncertainties are reported. The (red) vertical line and corresponding (gray) shaded column indicate the central value and the total uncertainty of the combined measurement, respectively. 
Figure 2(a) shows the invariant mass distribution of simulated $H \rightarrow \gamma \gamma$ events in two categories with one of the best and one of the worst experimental resolutions. Figure 2(b) shows the distribution of the data superimposed with the result of the simultaneous fit. The main sources of uncertainty include uncertainties in the linearity of the response due to the relative calibration of the different gains used in the calorimeter readout $(200 \mathrm{MeV})$, in the intercalibration of the different calorimeter layers $(190 \mathrm{MeV})$, in the knowledge of the material in front of the calorimeter $(160 \mathrm{MeV})$, in the modeling of the lateral shower shapes $(120 \mathrm{MeV})$ and the reconstruction of photon conversions $(50 \mathrm{MeV})$. The remaining uncertainties account for $85 \mathrm{MeV}$. Several checks are performed, which do not change the result significantly [10].

\section{Summary}

A measurement of the mass of the Higgs boson has been derived from a combined fit to the invariant mass spectra of the decay channels $H \rightarrow Z Z^{*} \rightarrow 4 \ell(\ell=e, \mu)$ and $H \rightarrow \gamma \gamma$ obtained from $p p$ collision date recorded by the ATLAS experiment at the CERN LHC at a centre-of-mass energy of $\sqrt{s}=13 \mathrm{TeV}$, corresponding to an integrated luminosity of $36.1 \mathrm{fb}^{-1}$. The measurements include, besides updates on the calibrations for muons, electrons, and photons, improved analysis techniques with respect to the previous result based on Run-1 data.

The measured values of the Higgs boson mass for the $H \rightarrow Z Z^{*} \rightarrow 4 \ell$ and $H \rightarrow \gamma \gamma$ decay modes are

$$
m_{H}=124.88 \pm 0.37 \text { (stat) } \pm 0.05 \text { (syst) } \mathrm{GeV}=124.88 \pm 0.37 \mathrm{GeV}
$$

and

$$
m_{H}=125.11 \pm 0.21 \text { (stat) } \pm 0.36 \text { (syst) } \mathrm{GeV}=125.11 \pm 0.42 \mathrm{GeV}
$$

respectively, and the mass derived from the combined fit is

$$
m_{H}=124.98 \pm 0.19 \text { (stat) } \pm 0.21 \text { (syst) } \mathrm{GeV}=124.98 \pm 0.28 \mathrm{GeV} .
$$

These results are in good agreement with the LHC Run-1 average of $125.09 \pm 0.24 \mathrm{GeV}$, as can be seen in Figure 3.

\section{References}

[1] ATLAS Collaboration, Observation of a new particle in the search for the Standard Model Higgs boson with the ATLAS detector at the LHC, Phys. Lett. B 716 (2012) 1, arXiv: 1207.7214 [hep-ex].

[2] CMS Collaboration,Observation of a new boson at a mass of $125 \mathrm{GeV}$ with the CMS experiment at the LHC, Phys. Lett. B 716 (2012) 30, arXiv: 1207.7235 [hep-ex].

[3] F. Englert and R. Brout, Broken Symmetry and the Mass of Gauge Vector Mesons, Phys. Rev. Lett. 13 (1964) 321.

[4] P. W. Higgs, Broken Symmetries and the Masses of Gauge Bosons, Phys. Rev. Lett. 13 (1964) 508.

[5] G. S Guralnik, C. R. Hagen and T. W. B. Kibble, Global Conservation Laws and Massless Particles, Phys. Rev. Lett. 13 (1964) 585. 
[6] ATLAS and CMS Collaborations, Combined Measurement of the Higgs Boson Mass in pp Collisions at $\sqrt{s}=7$ and 8 TeV with the ATLAS and CMS Experiments, Phys. Rev. Lett. 114 (2015) 191803, arXiv: 1503.07589 [hep-ex].

[7] ATLAS Collaboration, Measurement of the Higgs boson mass from the $H \rightarrow \gamma \gamma$ and $H \rightarrow Z Z^{*} \rightarrow 4 \ell$ channels in pp collisions at center-of-mass energies of 7 and $8 \mathrm{TeV}$ with the ATLAS detector, Phys. Rev. D 90 (2014) 052004, arXiv: 1406.3827 [hep-ex].

[8] CMS Collaboration, Precise determination of the mass of the Higgs boson and tests of compatibility of its couplings with the standard model predictions using proton collisions at 7 and 8 TeV, Eur. Phys. J. C 75 (2015) 212, arXiv: 1412.8662 [hep-ex].

[9] CMS Collaboration, Measurements of properties of the Higgs boson decaying into the four-lepton final state in pp collisions at $\sqrt{s}=13 \mathrm{TeV}$, JHEP 11 (2017) 047, arXiv: 1706.09936 [hep-ex].

[10] ATLAS Collaboration, Measurement of the Higgs boson mass in the $H \rightarrow Z Z^{*} \rightarrow 4 \ell$ and $H \rightarrow \gamma \gamma$ channels with $\sqrt{s}=13 \mathrm{TeV}$ p collisions using the ATLAS detector, ATLAS-CONF-2017-046, 2017, url: https://cds.cern.ch/record/2273853

[11] ATLAS Collaboration, The ATLAS Experiment at the CERN Large Hadron Collider, JINST 3 (2008) S08003.

[12] ATLAS Collaboration, Muon reconstruction performance of the ATLAS detector in proton-proton collision data at $\sqrt{s}=13$ TeV, Eur. Phys. J. C 76 (2016) 292, arXiv: 1603.05598 [hep-ex].

[13] ATLAS Collaboration, Measurement of the photon identification efficiencies with the ATLAS detector using LHC Run-1 data, Eur. Phys. J. C 76 (2016) 666, arXiv: 1606.01813 [hep-ex].

[14] ATLAS Collaboration, Measurement of inclusive and differential cross sections in the $H \rightarrow Z Z^{*} \rightarrow 4 \ell$ decay channel in pp collisions at $\sqrt{s}=13 \mathrm{TeV}$ with the ATLAS detector, JHEP 10 (2017) 132, arXiv: 1708.02810 [hep-ex].

[15] ATLAS Collaboration, Measurements of Higgs boson production and couplings in the four-lepton channel in pp collisions at center-of-mass energies of 7 and $8 \mathrm{TeV}$ with the ATLAS detector, Phys. Rev. D 91, 012006 (2015), arXiv: 1408.5191 [hep-ex].

[16] J. Alwall, R. Frederix, S. Frixione, V. Hirschi, F. Maltoni, O. Mattelaer, H.-S. Shao, T. Stelzer, P. Torrielli, M. Zaro, The automated computation of tree-level and next-to-leading order differential cross sections, and their matching to parton shower simulations, JHEP 07 (2014) 079, arXiv: 1405.0301 [hep-ph].

[17] S. Kullback and R. A. Leibler, On Information and Sufficiency, Ann. Math. Statist. 22 (1951) 79, url: http://dx.doi.org/10.1214/aoms/1177729694.

[18] C. Anastasiou, S. Buehler, F. Herzog and A. Lazopoulos, Inclusive Higgs boson cross-section for the LHC at 8 TeV, JHEP 04 (2012) 004, arXiv: 1202.3638 [hep-ph]. 